\title{
A Systematic Study of Magnetic Field in Relativistic Heavy-Ion Collisions in the RHIC and LHC Energy Regions
}

\author{
Yang Zhong, ${ }^{1,2}$ Chun-Bin Yang, ${ }^{1,3}$ Xu Cai, ${ }^{1,3}$ and Sheng-Qin Feng ${ }^{2,3}$ \\ ${ }^{1}$ Institute of Particle Physics, Central China Normal University, Wuhan 430079, China \\ ${ }^{2}$ Department of Physics, College of Science, China Three Gorges University, Yichang 443002, China \\ ${ }^{3}$ Key Laboratory of Quark and Lepton Physics (MOE), Central China Normal University, Wuhan 430079, China
}

Correspondence should be addressed to Yang Zhong; yzhong913@163.com

Received 26 June 2014; Accepted 13 August 2014; Published 26 August 2014

Academic Editor: Bhartendu K. Singh

Copyright (C) 2014 Yang Zhong et al. This is an open access article distributed under the Creative Commons Attribution License, which permits unrestricted use, distribution, and reproduction in any medium, provided the original work is properly cited. The publication of this article was funded by SCOAP ${ }^{3}$.

The features of magnetic field in relativistic heavy-ion collisions are systematically studied by using a modified magnetic field model in this paper. The features of magnetic field distributions in the central point are studied in the RHIC and LHC energy regions. We also predict the feature of magnetic fields at LHC $\sqrt{s_{N N}}=900,2760$, and $7000 \mathrm{GeV}$ based on the detailed study at RHIC $\sqrt{s_{N N}}=62.4,130$, and $200 \mathrm{GeV}$. The dependencies of the features of magnetic fields on the collision energies, centralities, and collision time are systematically investigated, respectively.

\section{Introduction}

Collisions of two heavy nuclei at high energy serve as a means for creating and exploring strongly interacting matter at high possible energy densities where a new extreme state of matter, the deconfined quark-gluon plasma (QGP), is expected to be formed [1-3]. Besides the formation of QGP, relativistic heavy-ion collisions create also extremely strong (electro)magnetic field due to the relativistic motion of the colliding heavy ions carrying large positive electric charge [36].

Now we turn to a key question: can the Chiral magnetic effect (CME) occur in heavy-ion collisions? The answer to the question seems to be positive. Two elements are needed for the CME to occur: an external magnetic field and a locally nonzero axial charge density. The relativistically moving heavy ions, typically with large positive charges (e.g., $+79 \mathrm{e}$, for $\mathrm{Au}$ ), carry strong magnetic (and electric) fields with them. In the short moments before/during/after the impact of two ions in noncentral collisions, there is a very strong magnetic field in the reaction zone [5-7]. In fact, such a magnetic field is estimated to be of the order of $m_{\pi}^{2} \approx 10^{18}$ Gauss [4, 8, 9], probably the strongest, albeit transient, magnetic field in the present universe. The other required element, a locally nonvanishing axial charge density, can also be created in the reaction zone during the collision process through sphaleron transitions (see, e.g., [10] for discussions and references therein). As such, it appears at least during the very early stage of a heavy-ion collision and there can be both strong magnetic field and nonzero axial charge density in the created hot matter.

It is suggested by $[5,6,11-13]$ that off-central heavyion collisions can create strong transient magnetic fields due to the fast, oppositely directed motion of two colliding ions. Thus, heavy-ion collisions provide a unique terrestrial environment to study QCD in strong magnetic fields [14-17]. It has been shown that a strong magnetic field can convert topological charge fluctuations in the QCD vacuum into global electric charge separation with respect to the reaction plane. This so-called Chiral magnetic effect may serve as a sign of the local P and CP violation of QCD.

Experimentally, the STAR [18-21], PHENIX [22], and ALICE [23] collaborations have presented the measurements of CME by the two-particle or three-particle correlations of charged particles with respect to the reaction plane, which are qualitatively consistent with the CME. A clear signal compatible with a charge dependent separation relative to the 
reaction plane is observed, which shows little or no collision energy dependence when compared to measurements at RHIC energies. This provides a new insight into understanding the nature of the charge-dependent azimuthal correlations observed at RHIC and LHC energies.

Kharzeev, Mclerran, and Warringa (KMW) [5] suggested that, in the vicinity of the deconfinement phase transition and under the influence of the strong magnetic field generated by the colliding nuclei, the quark spin alignment along the direction of the angular momentum and the imbalance of the left- and right-handed quarks generate an electromagnetic current. The experimental search of those effects has intensified recently, following the realization that the consequent quark fragmentation into charged hadrons results in a charge separation along the direction of the magnetic field and perpendicular to the reaction plane.

The magnitude of the effect either should not change or should decrease with increasing energy as long as a deconfined state of matter is formed in a heavy-ion collision. In addition, in $[24,25]$ it is also suggested that there should be no energy dependence between the top RHIC and the LHC energies, based on arguments related to the universality of the underlying physical process, without, however, explicitly quantifying what the contribution of the different values and time evolution of the magnetic field for different energies will be. On the other hand, it is argued [26] that the CME should strongly decrease at higher energies because the magnetic field decays more rapidly. Such a spread in the theoretical expectations makes it important to measure the chargedependent azimuthal correlations at the LHC, where the collision energy is an order of magnitude higher compared to the RHIC.

Charge separation needs a symmetry axis along which the separation can take place. The only symmetry axis in a heavy-ion collision is along the angular momentum which is perpendicular to the reaction plane. In central collisions there is no symmetry axis, so in that case charge separation should vanish. The strong magnetic field and the QCD vacuum can both completely be produced in the noncentral nucleusnucleus collision.

In [27], we used the Wood-Saxon nucleon distribution instead of a uniform one to improve the magnetic field calculation of the magnetic field for noncentral collision. It was argued that the magnitude of the magnetic field decreases at higher energies. The detailed research of the magnetic field dependencies on collision energies, impact parameter, and collision times is presented in this paper.

The paper is organized as follows. The improved calculation of magnetic field and the comparison of our new results with that given by KMW are described in Section 2, along with the predicted results of LHC energy region. A summary is given in Section 3.

\section{The Improved Calculation of Magnetic Field}

The situation with the experimental search for the local strong parity violation drastically changed once it was noticed [2830] that in noncentral nuclear collisions it would lead to the asymmetry in the emission of positively and negatively charged particle perpendicular to the reaction plane. Such a charge separation is a consequence of the difference in the number of quarks with positive and negative helicities positioned in the strong magnetic field of a noncentral nuclear collision, the so-called Chiral magnetic effect.

We begin with a charged particle moving along the direction of the $z$ axis. The magnetic field around it can be given by

$$
\vec{B}=\frac{1}{c^{2}} \vec{v} \times \vec{E}
$$

If the movement is relativistic, at the time $t=0$, the charge is the origin of the coordinate. The magnitude of the magnetic field $\vec{B}$ is given by

$$
B=\frac{1}{4 \pi \varepsilon_{0} c^{2}} \frac{q v\left(1-\beta^{2}\right) \sin \theta}{r^{2}\left(1-\beta^{2} \sin \theta\right)^{3 / 2}} .
$$

Now we consider a particle with charge $Z$ and rapidity $Y$ traveling along the $z$ axis. At $t=0$ the particle can be found at position $\vec{x}_{\perp}^{\prime}$; the magnetic field at the position $\vec{x}=\left(\vec{x}_{\perp}, z\right)$ caused by the particle is given by

$$
\begin{aligned}
e \vec{B}(\vec{x})= & Z \alpha_{\mathrm{EM}} \sinh Y \\
& \times \frac{\left(\vec{x}_{\perp}^{\prime}-\vec{x}_{\perp}\right) \times \vec{e}_{z}}{\left[\left(\vec{x}_{\perp}^{\prime}-\vec{x}_{\perp}\right)^{2}+(t \sinh Y-z \cosh Y)^{2}\right]^{3 / 2}} .
\end{aligned}
$$

Now we suppose that two similar nuclei with charge $Z$ and radius $R$ are traveling in the positive and negative $z$ direction with rapidity $Y_{0}$. At $t=0$ they have a noncentral collision with impact parameter $b$ at the origin point. We take the center of the two nuclei at $x= \pm b / 2$ at time $t=0$ so that the direction of $b$ lies along the $x$ axis.

As the nuclei are nearly traveling with the speed of light in typical heavy-ion collision experiments, the Lorentz contraction factor $\gamma$ is so large that we can consider the two included nuclei as pancake shaped. As a result, the nucleon's number density of each nucleus at $\vec{x}^{\prime}=\left(\vec{x}_{\perp}^{\prime}, z\right)$ can be given by

$$
\rho_{s \pm}\left(\vec{x}_{\perp}^{\prime}\right)=\frac{2}{4 / 3 \pi R^{3}} \sqrt{R^{2}-\left(\vec{x}_{\perp}^{\prime} \pm \frac{\vec{b}}{2}\right)^{2}}
$$

As a result, it seems that the nucleon distribution on average in a nucleus is an approximate result before considering the Lorentz contraction. In [1], KMW model used the uniform nuclear distribution as the nuclear distribution. But for a real situation, the nucleon distribution is not strictly uniform. It seems more reasonable to use the Wood-Saxon distribution in place of the uniform distribution. We use the Wood-Saxon distribution in this paper:

$$
n_{A}(r)=\frac{n_{0}}{1+\exp ((r-R) / d)} \text {, }
$$




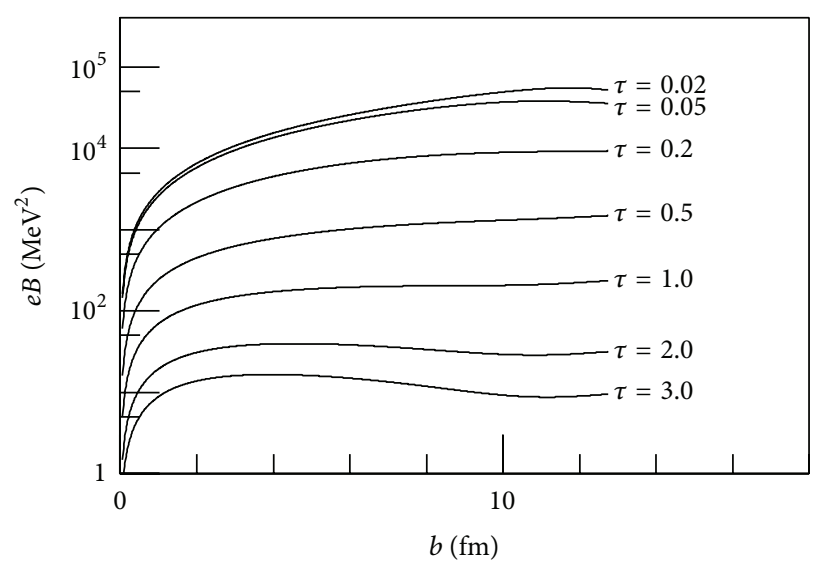

(a)

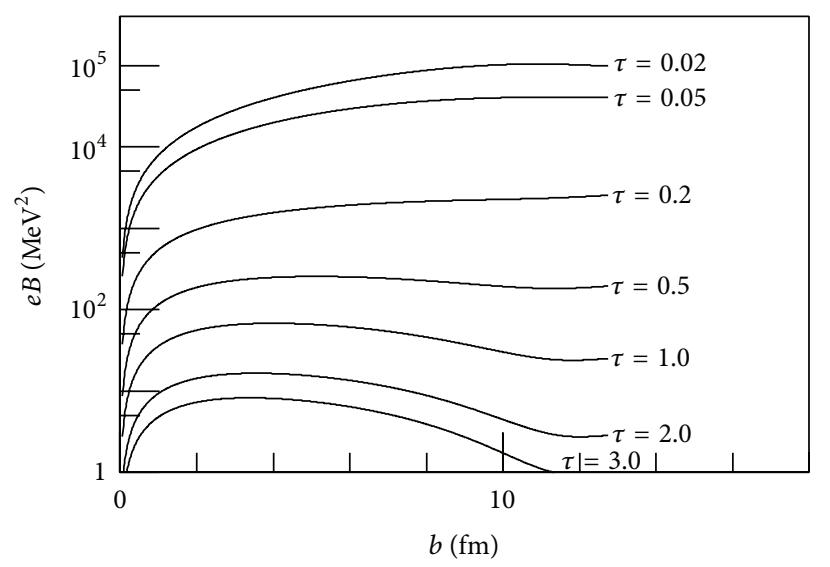

(c)

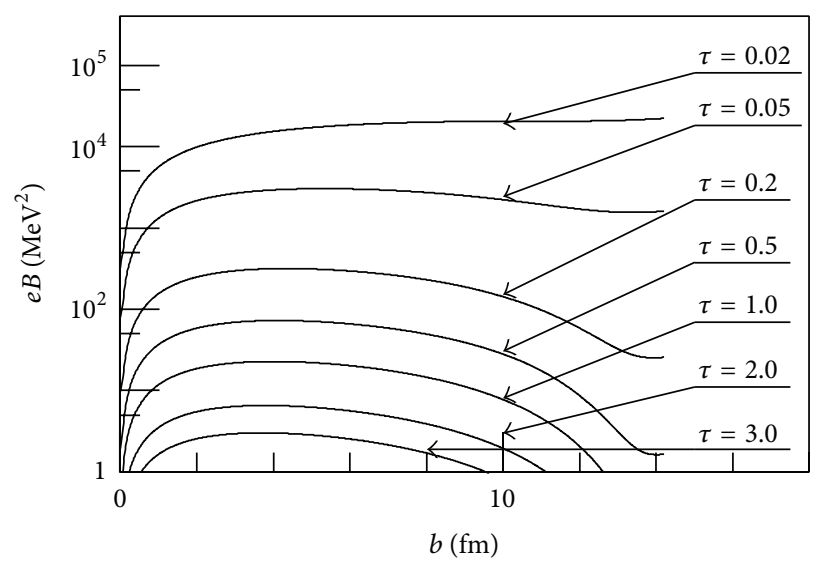

(e)

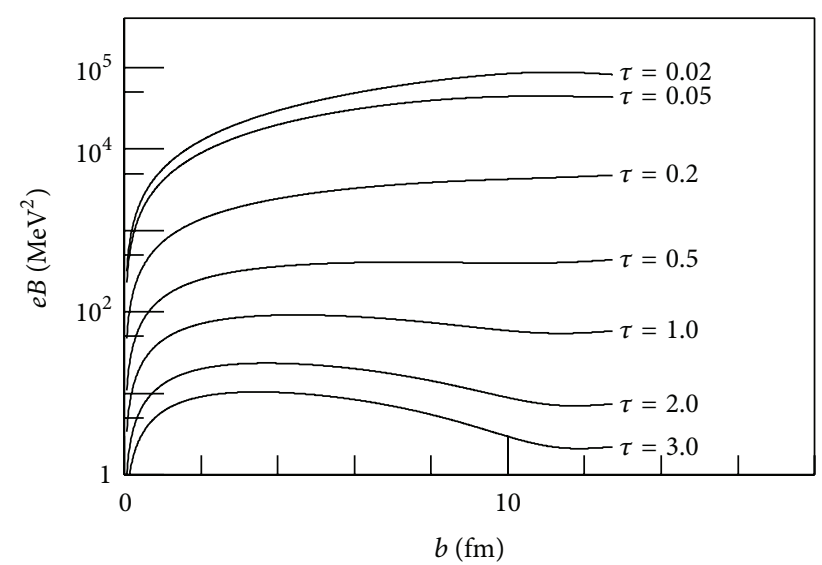

(b)

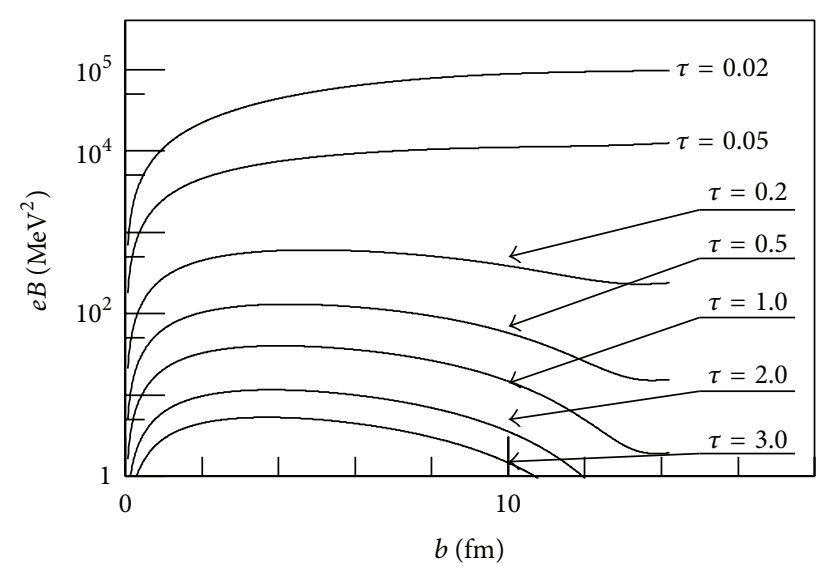

(d)

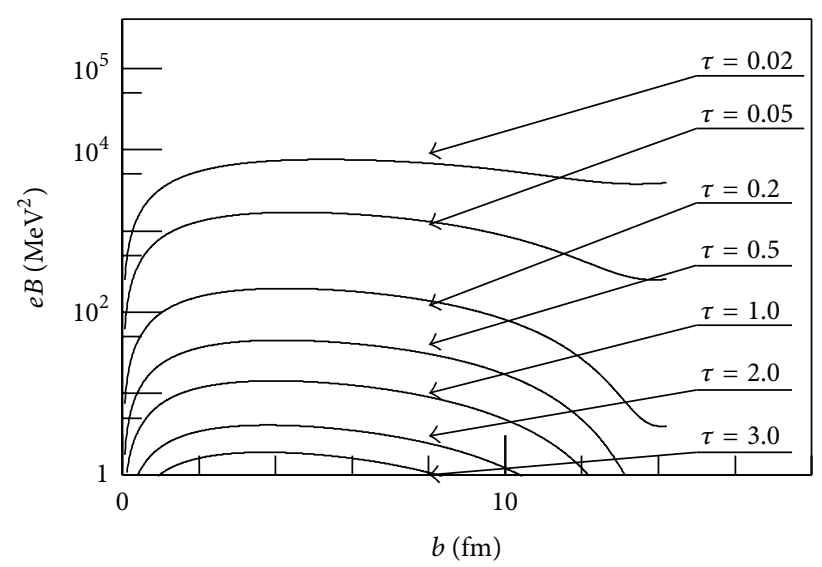

(f)

FIGURE 1: The dependencies of magnetic fields on impact parameters at different proper times $\tau$ at different energies of central mass system of $\sqrt{s_{N N}}=62.4 \mathrm{GeV}$ (a), $130 \mathrm{GeV}$ (b), $200 \mathrm{GeV}$ (c), $900 \mathrm{GeV}$ (d), $2760 \mathrm{GeV}$ (e), and $7000 \mathrm{GeV}$ (f), respectively. 


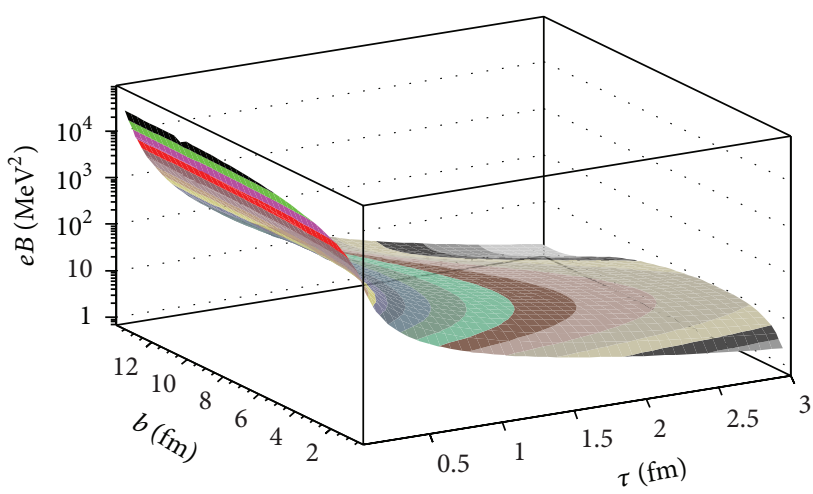

(a)

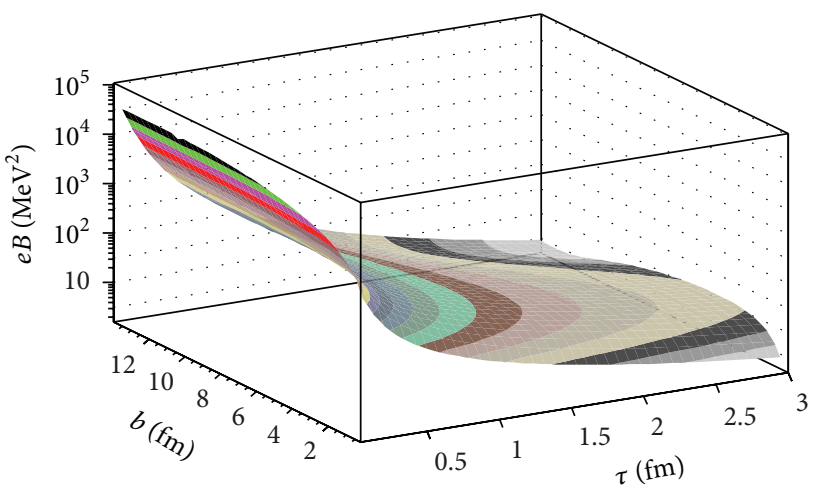

(b)

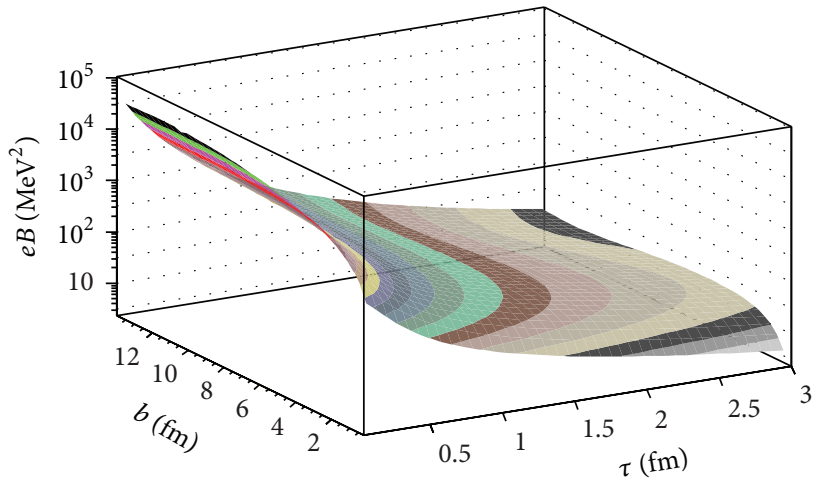

(c)

FIGURE 2: The dependencies of the magnetic field on proper time and impact parameters for Au-Au collisions at RHIC collision energies with $\sqrt{s}=200 \mathrm{GeV}(\mathrm{a}), \sqrt{s}=130 \mathrm{GeV}(\mathrm{b})$, and $\sqrt{s}=64 \mathrm{GeV}$ (c), respectively.

where $n_{0}=0.17 \mathrm{fm}^{-3}, d=0.54 \mathrm{fm}$, and the radius $R=$ $1.12 \mathrm{~A}^{1 / 3} \mathrm{fm}$. Considering the Lorentz contraction, the density in the two-dimensional plane can be given by

$$
\begin{aligned}
\rho_{ \pm}\left(\vec{x}_{\perp}^{\prime}\right)= & N \cdot \int_{-R}^{R} d z^{\prime} \\
& \times \frac{n_{0}}{1+\exp \left(\left(\sqrt{\left(x^{\prime} \mp b / 2\right)^{2}+y^{\prime 2}+z^{\prime 2}}-R\right) / d\right)},
\end{aligned}
$$

where $N$ is the normalization constant. The number densities should be normalized as

$$
\int d \vec{x}_{\perp}^{\prime} \rho_{ \pm}\left(\vec{x}_{\perp}^{\prime}\right)=1 .
$$

We now estimate the strength of the magnetic field at position $\vec{x}=\left(\vec{x}_{\perp}, z\right)$ caused by the two traveling nuclei. We are only interested in the time $t>0$, that is, just after the collision. Then we can split the contribution of particles to the magnetic field in the following way:

$$
\vec{B}=\vec{B}_{s}^{+}+\vec{B}_{s}^{-}+\vec{B}_{p}^{+}+\vec{B}_{p}^{-},
$$

where $\vec{B}_{s}^{ \pm}$and $\vec{B}_{p}^{ \pm}$are the contributions of the spectators and the participants moving in the positive or negative $z$ direction, respectively. For spectators, we assume that they do not scatter at all and that they keep travelling with the beam rapidity $Y_{0}$. According to (3), we use the density above and find

$$
\begin{aligned}
& e \vec{B}_{s}^{ \pm}\left(\tau, \eta, \vec{x}_{\perp}\right) \\
&= \pm Z \alpha_{\mathrm{EM}} \sinh \left(Y_{0} \mp \eta\right) \int d^{2} \vec{x}_{\perp}^{\prime} \rho_{ \pm}\left(\vec{x}_{\perp}^{\prime}\right) \\
& \times\left[1-\theta_{\mp}\left(\vec{x}_{\perp}^{\prime}\right)\right] \frac{\left(\vec{x}_{\perp}^{\prime}-\vec{x}_{\perp}\right) \times \vec{e}_{z}}{\left[\left(\vec{x}_{\perp}^{\prime}-\vec{x}_{\perp}\right)^{2}+\tau^{2} \sinh \left(Y_{0} \mp \eta\right)^{2}\right]^{3 / 2}},
\end{aligned}
$$

where $\tau=\left(t^{2}-z^{2}\right)^{1 / 2}$ is the proper time, $\eta=(1 / 2) \ln [(t$ $+z) /(t-z)]$ is the space-time rapidity, and

$$
\theta_{\mp}\left(\vec{x}_{\perp}^{\prime}\right)=\theta\left[R^{2}-\left(\vec{x}_{\perp}^{\prime} \pm \frac{\vec{b}}{2}\right)^{2}\right] .
$$

Here, we would like to neglect the contribution from the particles created by the interactions and so we just need to take into account the contribution of the participants that were originally there. The distribution of participants that remain travelling along the beam axis is given by

$$
f(Y)=\frac{a}{2 \sinh \left(a Y_{0}\right)} e^{a Y}, \quad-Y_{0} \leq Y \leq Y_{0} .
$$




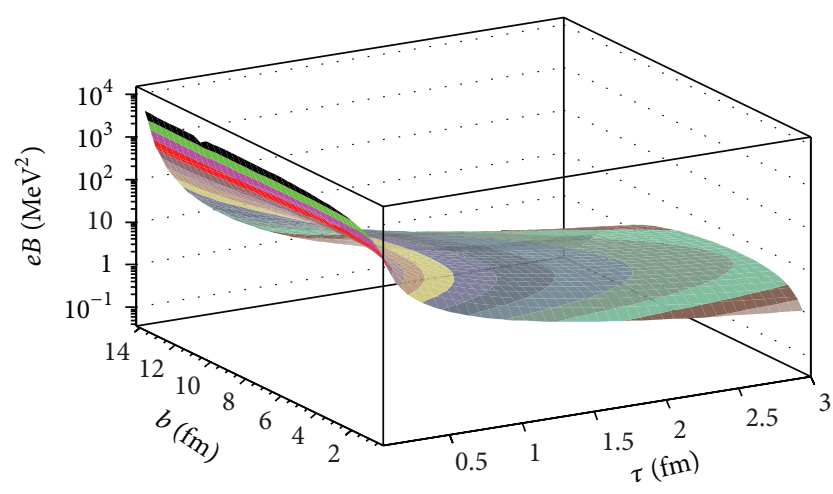

(a)

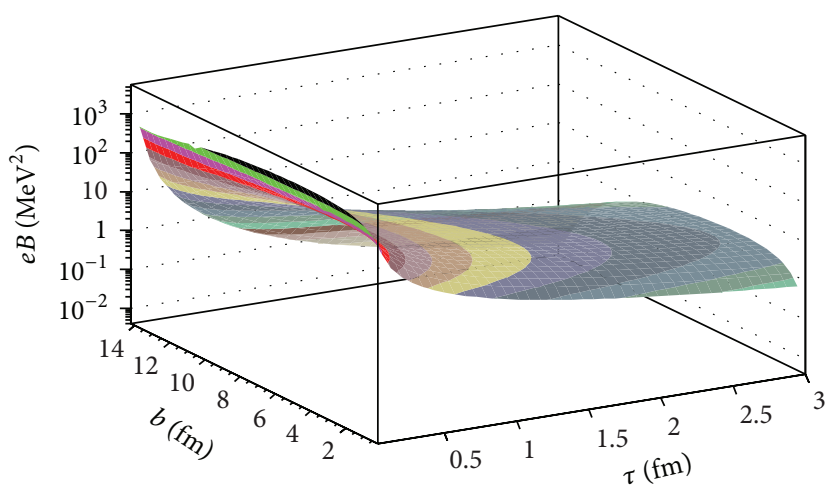

(b)

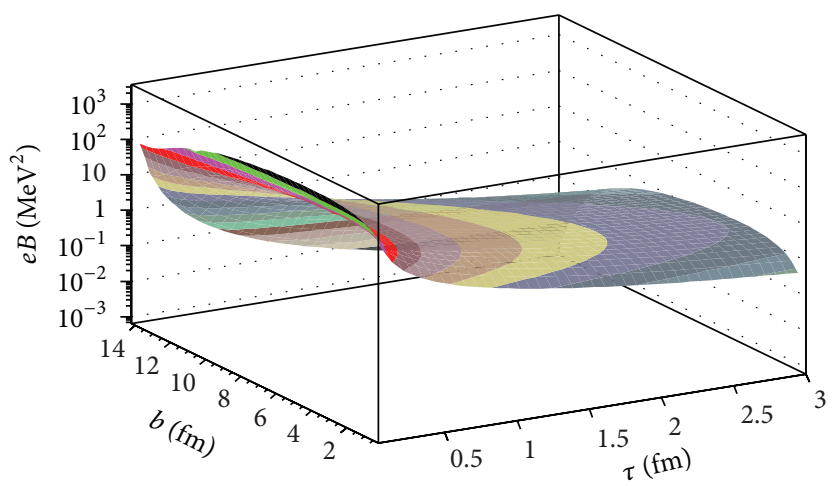

(c)

Figure 3: The dependencies of the magnetic field on proper time and impact parameters for $\mathrm{Pb}-\mathrm{Pb}$ collisions at LHC collision energies with $\sqrt{s}=900 \mathrm{GeV}(\mathrm{a}), \sqrt{s}=2760 \mathrm{GeV}(\mathrm{b})$, and $\sqrt{s}=7000 \mathrm{GeV}$ (c), respectively.

Experimental data shows that $a \approx 1 / 2$, consistent with the baryon junction stopping mechanism. The contribution of the participants to the magnetic field can be also given by

$$
\begin{aligned}
e \vec{B}_{p}^{ \pm}\left(\tau, \eta, \vec{x}_{\perp}\right) \\
= \pm Z \alpha_{\mathrm{EM}} \int d^{2} \vec{x}_{\perp}^{\prime} \int d Y f(Y) \sinh (Y \mp \eta) \\
\quad \times \rho_{ \pm}\left(\vec{x}_{\perp}^{\prime}\right) \theta_{\mp}\left(\vec{x}_{\perp}^{\prime}\right) \frac{\left(\vec{x}_{\perp}^{\prime}-\vec{x}_{\perp}\right) \times \vec{e}_{z}}{\left[\left(\vec{x}_{\perp}^{\prime}-\vec{x}_{\perp}\right)^{2}+\tau^{2} \sinh (Y \mp \eta)^{2}\right]^{3 / 2}} .
\end{aligned}
$$

We calculate the magnetite of the magnetic field at the origin $\left(\eta=0, \vec{x}_{\perp}=0\right)$ in which case it is pointing in the $y$ direction. We took a Au-Au collision with different beam rapidity and different impact parameters.

Figure 1 shows that the dependencies of magnetic fields of the central point on impact parameters at different proper times $\tau$ at different center-of-mass energies for RHIC and LHC, respectively. Figures $1(\mathrm{a}), 1(\mathrm{~b})$, and 1(c) are for the results in RHIC energy region. It is shown from Figures 1(a), 1(b), and 1(c) that the magnitudes of the magnetic fields increase with the increasing impact parameter ay proper time $\tau \leq 1.0 \mathrm{fm}$. Figures $1(\mathrm{~d}), 1(\mathrm{e})$, and $1(\mathrm{f})$ are for the results in LHC energy region. Figures $1(\mathrm{~d})$ and $1(\mathrm{e})$ show that the magnitudes of the magnetic field increase with the increasing impact parameter only at proper time $\tau \leq 0.05 \mathrm{fm}$ but start to decrease with increasing impact parameter when proper time $\tau>0.05 \mathrm{fm}$. Figure $1(\mathrm{f})$ shows that the magnitudes of the magnetic field decrease with the increasing impact parameter at proper time $\tau \geq 0.02 \mathrm{fm}$ at $\sqrt{s_{N N}}=7000 \mathrm{GeV}$. In comparison with that of RHIC energy region, we find that the magnitudes of the magnetic fields of $\tau \geq 2 \mathrm{fm}$ fall to zero more rapidly at LHC energy region. The variation characteristics of magnetic field with impact parameter at RHIC energy region are different from that of LHC energy region.

The dependencies of the magnetic field on proper time and impact parameters for Au-Au collisions at RHIC energy region with $\sqrt{s}=200 \mathrm{GeV}, \sqrt{s}=130 \mathrm{GeV}$, and $\sqrt{s}=64 \mathrm{GeV}$ are shown in Figures 2(a), 2(b), and 2(c), respectively. The same tendency of the dependencies of the magnetic field on proper time and impact parameters at RHIC is observed from Figure 2. The maximum position of magnetic field is located at much smaller proper time $\tau \sim 0.02 \mathrm{fm}$ and much larger impact parameter $b \simeq 12 \mathrm{fm}$ shown in Figure 2 . It is observed that the magnitude of magnetic field decreases sharply with increasing proper time $\tau$ and decreases with decreasing impact parameter $b$.

Figures 3(a), 3(b), and 3(c) show that the maximum of magnetic field decrease with the increase of collision energies when $\sqrt{s}$ from $900 \mathrm{GeV}$ to $7000 \mathrm{GeV}$ at LHC energy region. It is found that the maximum of magnetic field are about $3.0 \times 10^{3} \mathrm{MeV}^{2}, 4.0 \times 10^{2} \mathrm{MeV}^{2}$, and $80.0 \mathrm{MeV}^{2}$ at $\sqrt{s}=900$, 


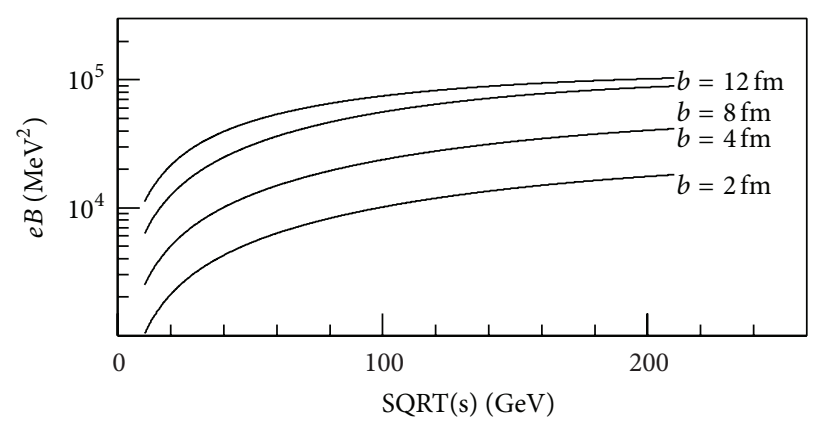

(a)

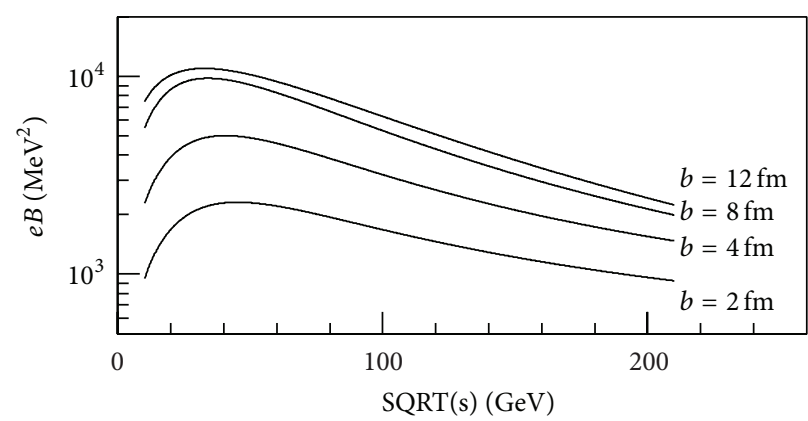

(c)

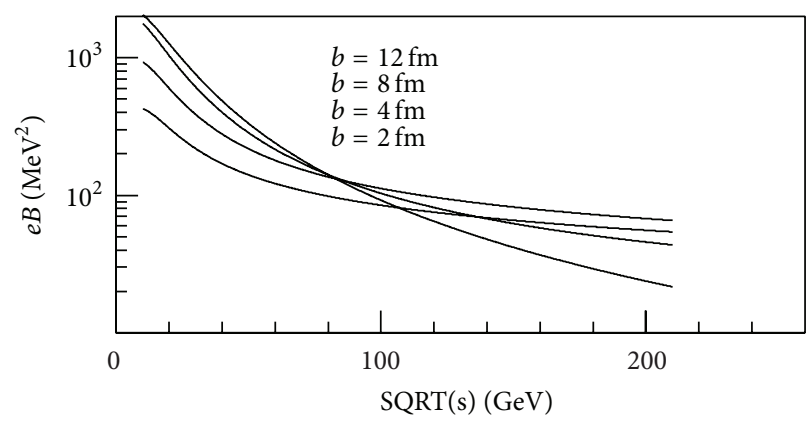

(e)

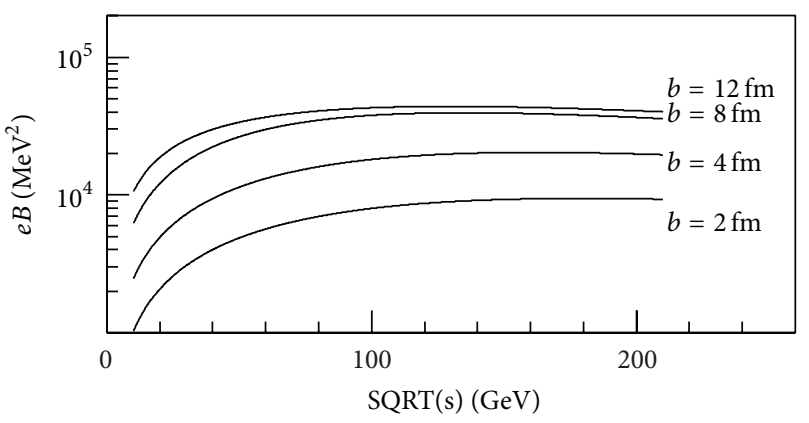

(b)

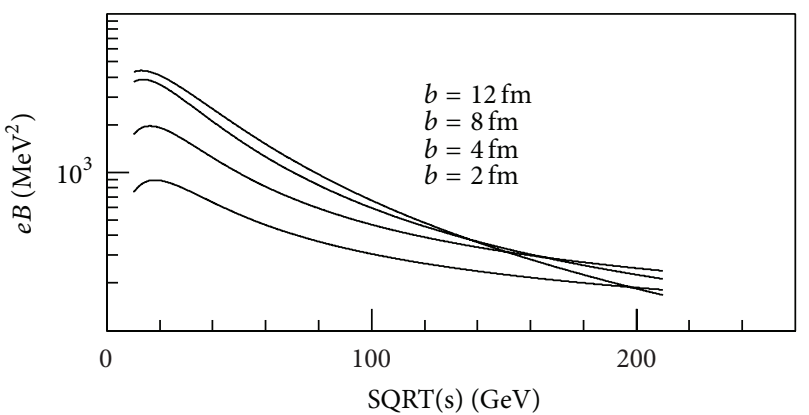

(d)

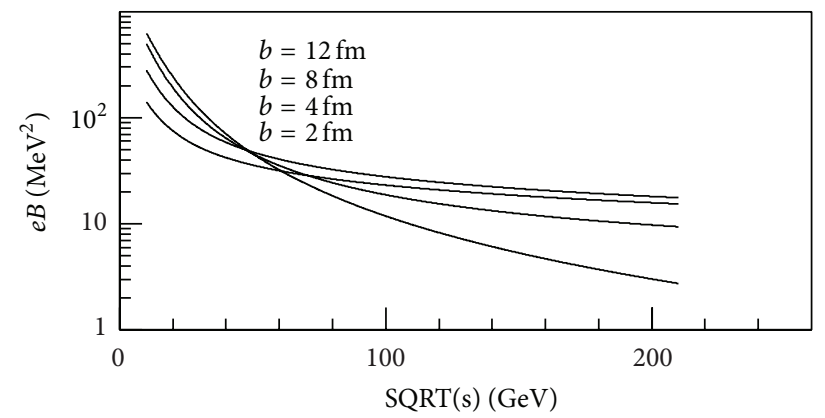

(f)

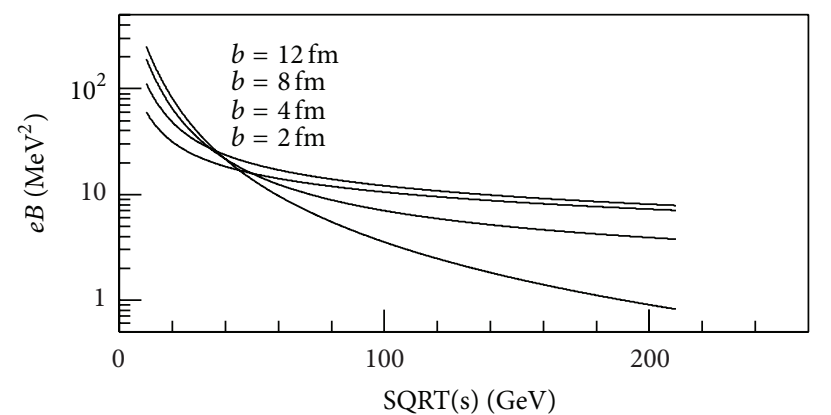

(g)

FIGURE 4: The dependencies of the magnetic field on collision energies of central mass system less than $200 \mathrm{GeV}$ at RHIC energy region at different impact parameters for Au-Au collisions at different proper times for $\tau=0.02 \mathrm{fm}(\mathrm{a}), \tau=0.05 \mathrm{fm}(\mathrm{b}), \tau=0.2 \mathrm{fm}(\mathrm{c}), \tau=0.5 \mathrm{fm}(\mathrm{d})$, $\tau=1.0 \mathrm{fm}(\mathrm{e}), \tau=2.0 \mathrm{fm}(\mathrm{f})$, and $\tau=3.0 \mathrm{fm}$ (g), respectively. 


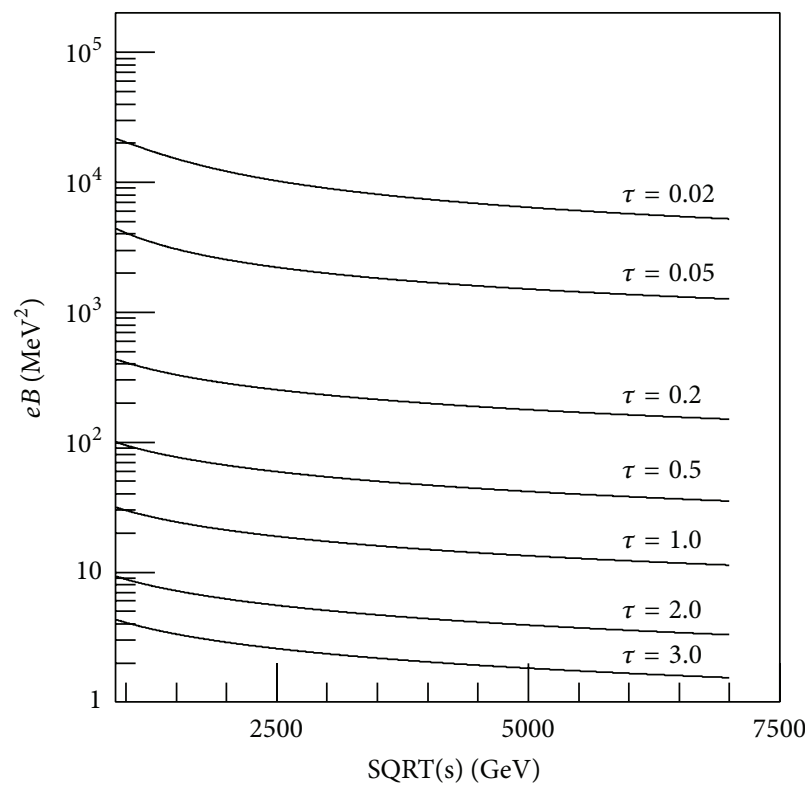

(a)

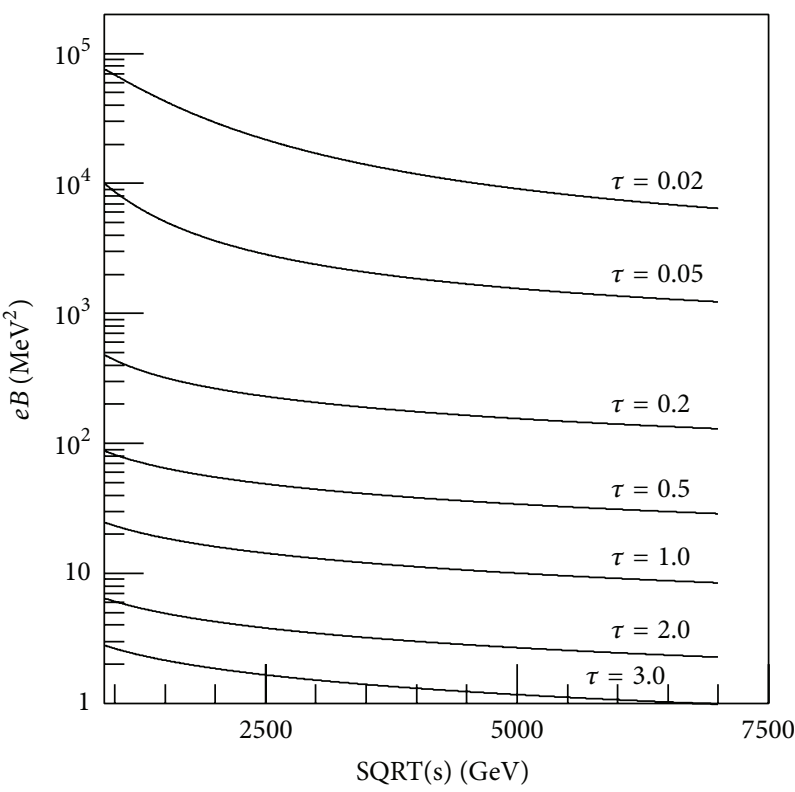

(c)

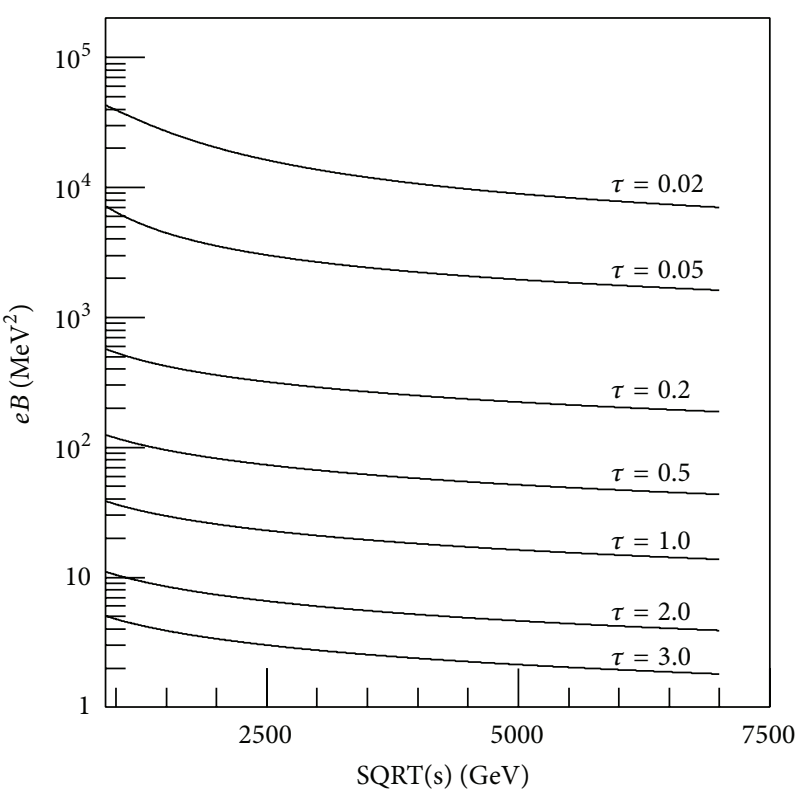

(b)

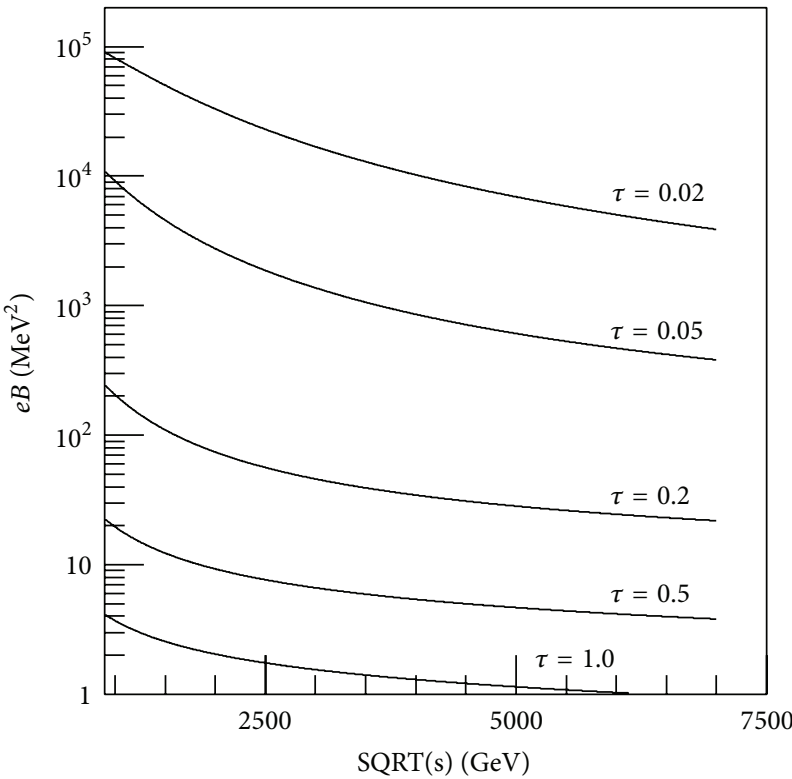

(d)

FIGURE 5: The dependencies of the magnetic field on collision energies of central mass system of LHC energy region of $\mathrm{Pb}-\mathrm{Pb}$ collisions at different proper times at different impact parameters for $b=2 \mathrm{fm}(\mathrm{a}), b=4 \mathrm{fm}(\mathrm{b}), b=8 \mathrm{fm}(\mathrm{c})$, and $b=12 \mathrm{fm}$ (d), respectively.

$\sqrt{s}=2760$, and $\sqrt{s}=7000 \mathrm{GeV}$, respectively. These are much smaller than that in RHIC energy region. The same variation characteristics of magnetic field with impact parameter and proper time are observed at LHC energy region and that of RHIC.

The dependencies of the magnetic field on collision energies $\sqrt{s}$ at RHIC energy region at different impact parameters for Au-Au collisions at different proper time $\tau$ are shown in Figure 4. It is found that the dependencies of magnetic field on collision energies at different impact parameter are all on the rising trend at $\tau=0.02 \mathrm{fm}$ and $\tau=0.05 \mathrm{fm}$ as shown in
Figures 4(a) and 4(b). Figure 4(c) $(\tau=0.2 \mathrm{fm})$ shows that the magnetic fields increase with the increase of collision energies when $\sqrt{s} \leq 30 \mathrm{GeV}$ but then decrease with the increase of collision energies when $\sqrt{s}>30 \mathrm{GeV}$. As the proper time $\tau$ increases to $\tau \geq 0.5$ as shown in Figures 4(d), 4(e), 4(f), and $4(\mathrm{~g})$, the magnetic fields decrease with the increase of collision energies and decrease more rapidly of more offcentral collisions of $b=12$ than the more central collisions of $b=2$ and $4 \mathrm{fm}$.

Figure 5 shows that the dependencies of the magnetic field on collision energies of central mass system of LHC 


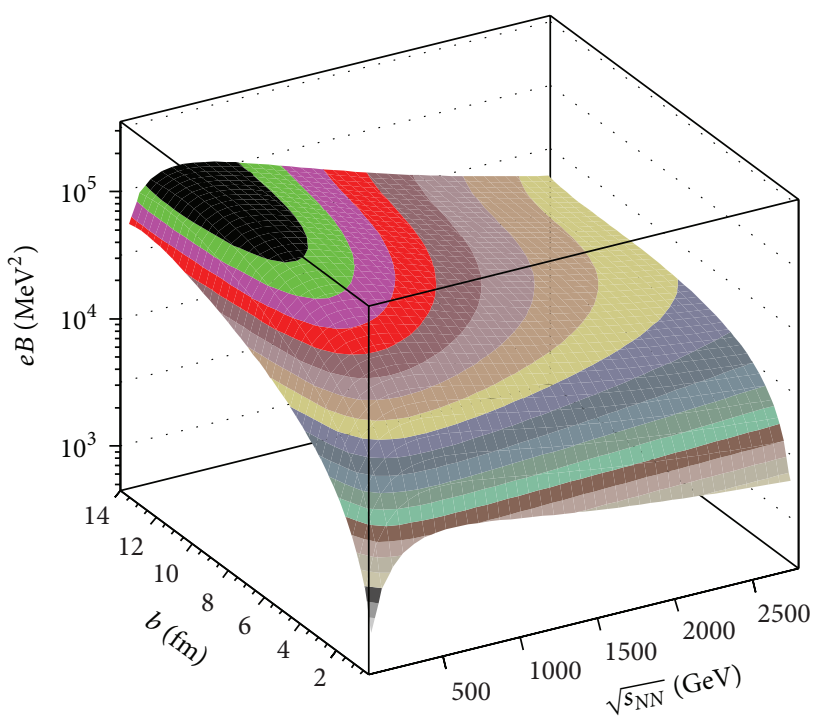

(a)

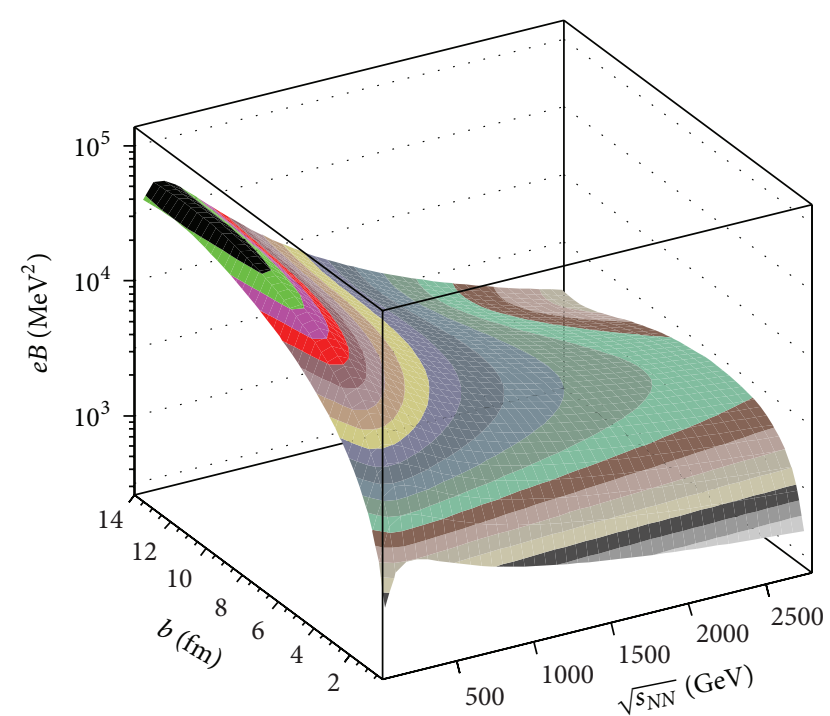

(b)

Figure 6: The dependencies of the magnetic field on collision energies of central mass system and impact parameters at different proper times for $\tau=0.02 \mathrm{fm}$ (a) and $\tau=0.05 \mathrm{fm}$ (b).

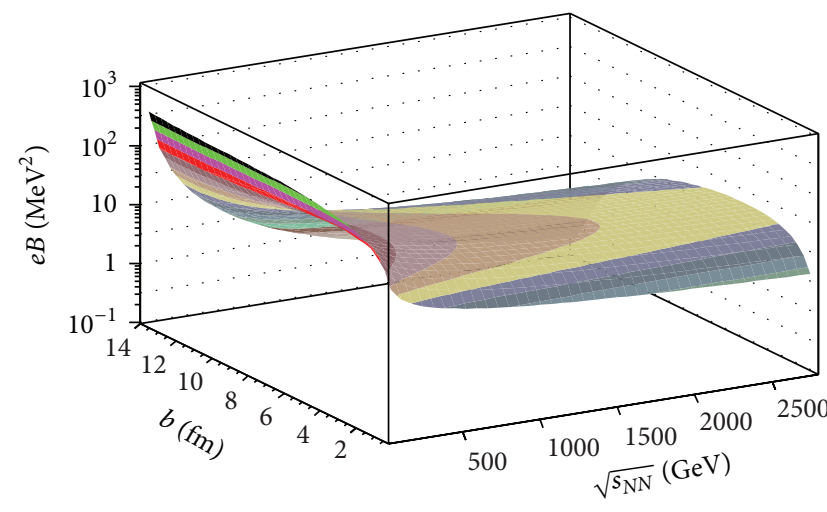

(a)

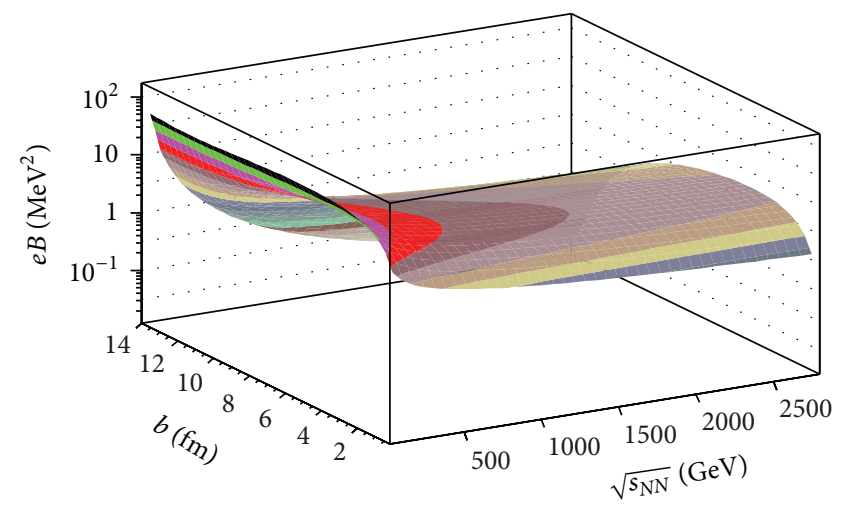

(b)

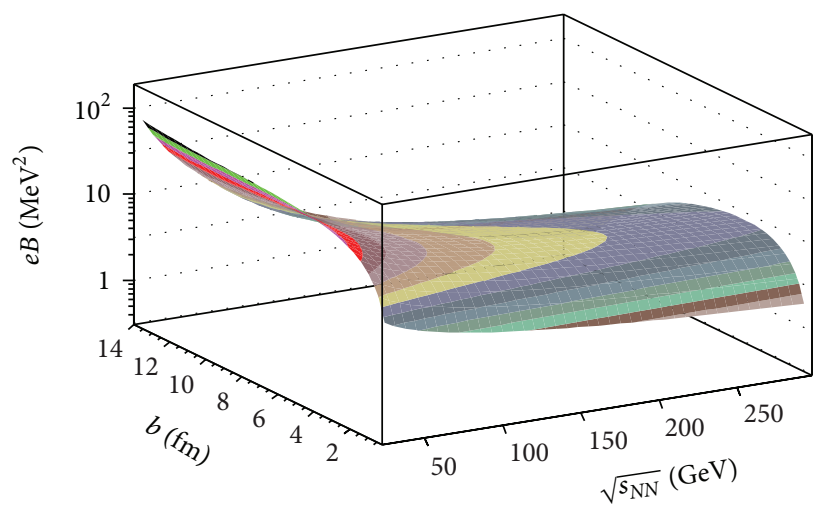

(c)

FIGURE 7: The dependencies of the magnetic field on collision energies of central mass system and impact parameters at different proper times for $\tau=1.0 \mathrm{fm}(\mathrm{a}), \tau=2.0 \mathrm{fm}(\mathrm{b})$, and $\tau=3.0 \mathrm{fm}(\mathrm{c})$, respectively. 
energy region of $\mathrm{Pb}-\mathrm{Pb}$ collisions at different proper times at different impact parameters for $b=2 \mathrm{fm}, b=4 \mathrm{fm}, b=8 \mathrm{fm}$, and $b=12 \mathrm{fm}$ with different proper time $\tau$. It is found that the magnitudes of magnetic fields decrease with increasing energy at LHC energy region, and the speed of decreasing at large impact parameter such as $b=12 \mathrm{fm}$ (shown in Figure 5(d)) is more quick than that of more central collision such as $b=2 \mathrm{fm}$ (shown in Figure 5(a)). The magnetic field approaches zero as $\tau \geq 1.0 \mathrm{fm}$ when $b=12 \mathrm{fm}$.

The dependencies of the magnetic field on collision energies of central mass system and impact parameters at different proper times for $\tau=0.02 \mathrm{fm}, 0.05 \mathrm{fm}, 1.0 \mathrm{fm}, 2.0 \mathrm{fm}$, and $3.0 \mathrm{fm}$ are shown in Figures 6 and 7, respectively. The threedimensional schemes of different proper time show that the large magnetic fields are produced during small $\tau$, large impact parameter, and $\sqrt{\left(s_{N N}\right)} \leq 500 \mathrm{GeV}$ energy regions. The magnetic fields decrease with increasing collision energy

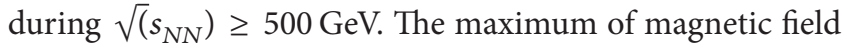
is $e B \simeq 50 \mathrm{MeV}^{2}$ when $\tau=2.0 \mathrm{fm}$, but that of $\tau=0.02 \mathrm{fm}$ is $e B \simeq 10^{5} \mathrm{MeV}^{2}$.

\section{Summary and Conclusion}

It is found that the relativistically moving heavy ions, typically with large positive charges, carry strong magnetic (and electric) fields with them. In the short moments before/during/ after the impact of two ions in noncentral collisions, there is a very strong magnetic field in the reaction zone. In fact, such a magnetic field is estimated to be of the order of $m_{\pi}^{2} \approx 10^{18}$ Gauss, probably the strongest magnetic field in the present universe. It appears at least during the very early stage of a heavy-ion collision and there can be both strong magnetic field and nonzero axial charge density in the created hot matter.

It is suggested that off-central heavy-ion collisions can create strong transient magnetic fields due to the fast, oppositely directed motion of two colliding ions. Thus, a unique terrestrial environment to study QCD in strong magnetic fields is provided in relativistic heavy-ion collisions. A strong magnetic field can convert topological charge fluctuations in the QCD vacuum into global electric charge separation with respect to the reaction plane. This so-called Chiral magnetic effect may serve as a sign of the local $\mathrm{P}$ and $\mathrm{CP}$ violation of QCD.

The features of magnetic fields at LHC $\sqrt{s_{N N}}=900,2760$, and $7000 \mathrm{GeV}$ and at RHIC $\sqrt{s_{N N}}=62.4,130$, and $200 \mathrm{GeV}$ are systematically discussed. The dependencies of the features of magnetic fields on the collision energies, centralities, and collision time are systematically investigated, respectively.

We show that an enormous magnetic field can indeed be created in off-central heavy-ion collisions. The magnitude of the field is quite large, especially just after the collision, and decreases rapidly with time. The drop velocity increases with the collision energy increase. It is shown that the magnitudes of magnetic fields decrease with increasing energy at LHC energy region, and the speed of decreasing at large impact parameter such as $b=12 \mathrm{fm}$ is more quick than that of more central collision such as $b=2 \mathrm{fm}$.
The dependencies of the magnetic field on proper time and impact parameters for at RHIC and LHC energy regions, respectively. In comparison with that of RHIC energy region, we find that the magnitudes of the magnetic fields with proper time fall more rapidly at LHC energy region. The variation characteristics of magnetic field with impact parameter at RHIC energy region are different from that of LHC energy region. The maximum position is located in the small proper time $(\tau \sim 0.02 \mathrm{fm})$, more off-central collisions of $(b \simeq 12 \mathrm{fm})$, and $\sqrt{s_{N N}} \sim 200 \mathrm{GeV}$. The maximum of magnetic field in our calculation is about $e B \simeq 10^{5} \mathrm{MeV}^{2}$ when $\tau=0.02, b \simeq 12 \mathrm{fm}$, and $\sqrt{s_{N N}} \sim 200 \mathrm{GeV}$.

When surveying the dependencies of magnetic field on the proper time $\tau$, we find that the magnitude of the magnetic field decreases with the increasing energy when $\sqrt{s_{N N}} \geq 200 \mathrm{GeV}$. The systematic research of magnetic field is consistent with the suggestion given by $[24,25]$ that the Chiral magnetic effect either should not change or should decrease with increasing energy as long as a deconfined state of matter is formed in a heavy-ion collision.

\section{Conflict of Interests}

The authors declare that there is no conflict of interests regarding the publication of this paper.

\section{Acknowledgments}

This work was supported in part by National Natural Science Foundation of China (Grant nos. 11375069, 11175070, 11075061, 10975091, and 11221504), by the Ministry of Education of China under Grant no. 306022, by the Programme of Introducing Talents of Discipline to Universities under Grant no. B08033, and by Key Laboratory foundation of Quark and Lepton Physics (Central China Normal University) (QLPL2014P01).

\section{References}

[1] T. D. Lee, "A theory of spontaneous t violation," Physical Review $D$, vol. 8, no. 4, Article ID 1226, 14 pages, 1973.

[2] T. D. Lee and G. C. Wick, "Vacuum stability and vacuum excitation in a spin-0 field theory," Physical Review D, vol. 9, article 2291, 26 pages, 1974.

[3] H. Minakata and B. Müller, "Disoriented chiral condensate and strong electromagnetic fields," Physics Letters B, vol. 377, no. 1-3, pp. 135-139, 1996.

[4] V. V. Skokov, A. Y. Illarionov, and V. D. Toneev, "Estimate of the magnetic field strength in heavy-ion collisions," International Journal of Modern Physics A, vol. 24, no. 31, pp. 5925-5932, 2009.

[5] D. E. Kharzeev, L. D. McLerran, and H. J. Warringa, "The effects of topological charge change in heavy ion collisions: 'Event by event P and CP violation," Nuclear Physics A, vol. 803, no. 3-4, pp. 227-253, 2008.

[6] K. Fukushima, D. E. Kharzeev, and H. J. Warringa, "Real-time dynamics of the chiral magnetic effect," Physical Review Letters, vol. 104, no. 21, Article ID 212001, 2010.

[7] J. Rafelski and B. Müller, "Magnetic splitting of quasimolecular electronic states in strong fields," Physical Review Letters, vol. 36, no. 10, pp. 517-520, 1976. 
[8] A. Bzdak and V. Skokov, "Event-by-event fluctuations of magnetic and electric fields in heavy ion collisions," Physics Letters B: Nuclear, Elementary Particle and High-Energy Physics, vol. 710, no. 1, pp. 171-174, 2012.

[9] W. Deng and X. Huang, "Event-by-event generation of electromagnetic fields in heavy-ion collisions," Physical Review C, vol. 85, no. 4, Article ID 044907, 17 pages, 2012.

[10] D. E. Kharzeev, “Topologically induced local $P$ and $C P$ violation in QCD × QED," Annals of Physics, vol. 325, no. 1, pp. 205-218, 2010.

[11] K. Fukushima, D. E. Kharzeev, and H. J. Warringa, "Electriccurrent susceptibility and the Chiral Magnetic Effect," Nuclear Physics A, vol. 836, no. 3-4, pp. 311-336, 2010.

[12] H. J. Warringa, "Dynamics of the chiral magnetic effect in a weak magnetic field," Physical Review D, vol. 86, no. 8, Article ID 085029, 2012.

[13] D. Kharzeev, "Parity violation in hot QCD: why it can happen, and how to look for it," Physics Letters B, vol. 633, no. 2-3, pp. 260-264, 2006.

[14] D. Kharzeev and A. Zhitnitsky, "Charge separation induced by P-odd bubbles in QCD matter," Nuclear Physics A, vol. 797, no. 1-2, pp. 67-79, 2007.

[15] K. Fukushima, D. E. Kharzeev, and H. J. Warringa, "Chiral magnetic effect," Physical Review D, vol. 78, no. 7, Article ID 074033, 2008.

[16] Y. Burnier, D. E. Kharzeev, J. Liao, and H. Yee, "Chiral magnetic wave at finite baryon density and the electric quadrupole moment of the quark-gluon plasma," Physical Review Letters, vol. 107, Article ID 052303, 4 pages, 2011.

[17] D. E. Kharzeev and D. T. Son, "Testing the chiral magnetic and chiral vortical effects in heavy ion collisions," Physical Review Letters, vol. 106, no. 6, Article ID 062301, 4 pages, 2011.

[18] B. I. Abelev, M. M. Aggarwal, Z. Ahammed et al., "Azimuthal charged-particle correlations and possible local strong parity violation," Physical Review Letters, vol. 103, Article ID 251601, 6 pages, 2009.

[19] B. I. Abelev, M. M. Aggarwal, and Z. Ahammed, "Observation of charge-dependent azimuthal correlations and possible local strong parity violation in heavy ion collisions," Physical Review C, vol. 81, Article ID 054908, 17 pages, 2010.

[20] I. V. Selyuzhenkov and STAR Collaboration, "Global polarization and parity violation study in $\mathrm{Au}+\mathrm{Au}$ collisions," Romanian Reports in Physics, vol. 58, no. 1, pp. 49-53, 2006.

[21] S. A. Voloshin, "Experimental study of local strong parity violation in relativistic nuclear collisions," Nuclear Physics A, vol. 830, no. 1-4, pp. 377c-384c, 2009.

[22] N. N. Ajitanand, R. A. Lacey, A. Taranenko, and J. M. Alexander, "New method for the experimental study of topological effects in the quark-gluon plasma," Physical Review C, vol. 83, Article ID 011901, 6 pages, 2011.

[23] B. Abelev, J. Adam, D. Adamova et al., "Charge separation relative to the reaction plane in $\mathrm{Pb}-\mathrm{Pb}$ collisions at $\sqrt{s_{N N}}=2.76$ TeV," Physical Review Letters, vol. 110, Article ID 01230, 11 pages, 2013.

[24] A. R. Zhitnitsky, "P and CP violation and new thermalization scenario in heavy ion collisions," Nuclear Physics A, vol. 853, no. 1, pp. 135-163, 2011.

[25] A. R. Zhitnitsky, "Local P violation effects and thermalization in QCD: views from quantum field theory and holography," Nuclear Physics A, vol. 886, pp. 17-47, 2012.
[26] V. D. Toneev and V. Voronyuk, "Beam-energy and system-size dependence of the CME," Physics of Particles and Nuclei Letters, vol. 8, no. 9, pp. 938-943, 2011.

[27] Y. J. Mo, S. Q. Feng, and Y. F. Shi, "Effect of the Wood-Saxon nucleon distribution on the chiral magnetic field in relativistic heavy-ion collisions," Physical Review C, vol. 88, Article ID 024901, 8 pages, 2013.

[28] S. A. Voloshin, "Testing the chiral magnetic effect with central U+U collisions," Physical Review Letters, vol. 105, no. 17, Article ID 172301, 5 pages, 2010.

[29] F. Wang, "Effects of cluster particle correlations on local parity violation observables," Physical Review C, vol. 81, Article ID 064902, 11 pages, 2010.

[30] M. Asakawa, A. Majumder, and B. Müller, "Electric charge separation in strong transient magnetic fields," Physical Review CNuclear Physics, vol. 81, Article ID 064912, 20 pages, 2010. 

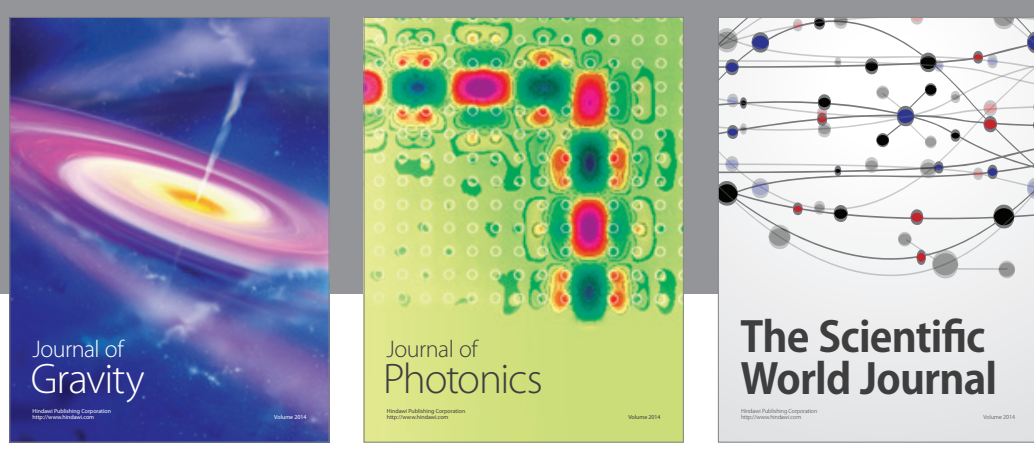

The Scientific World Journal
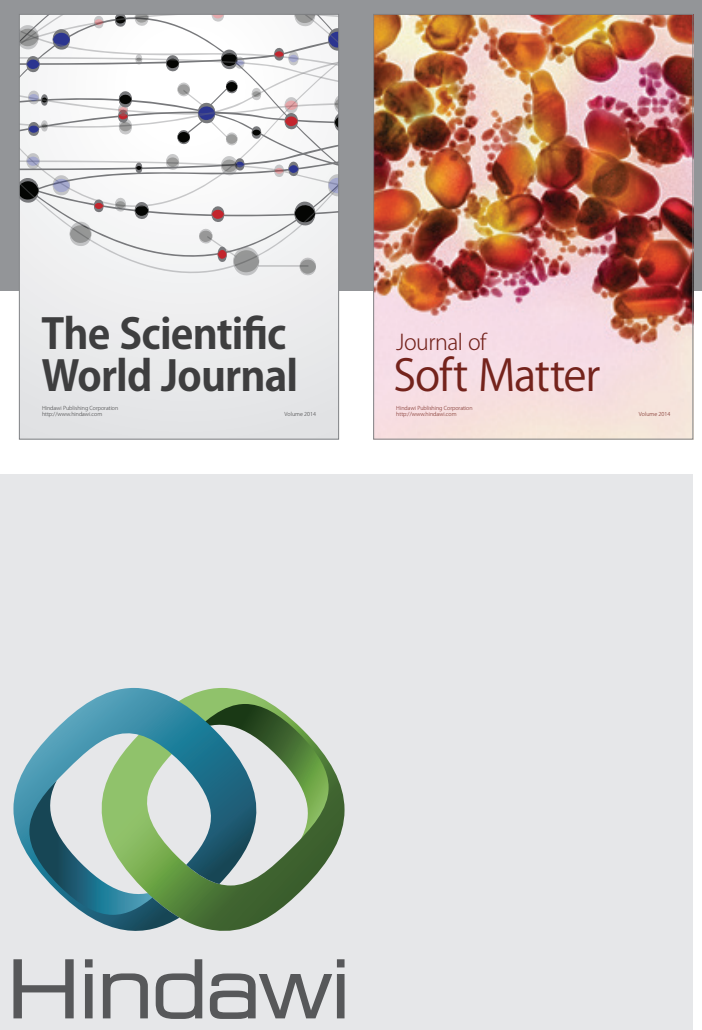

Submit your manuscripts at

http://www.hindawi.com

nternational Journal of

Statistical Mechanics
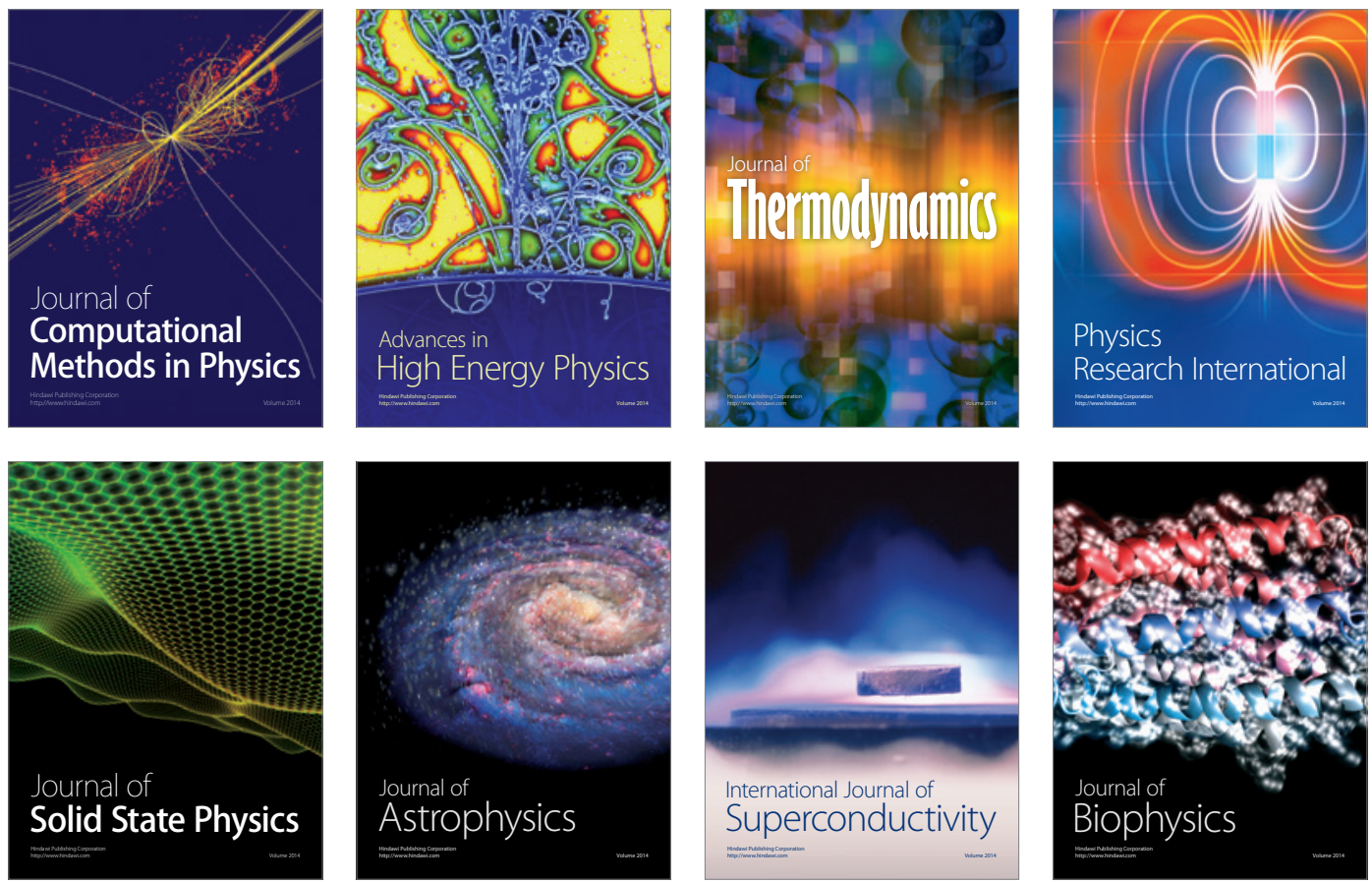
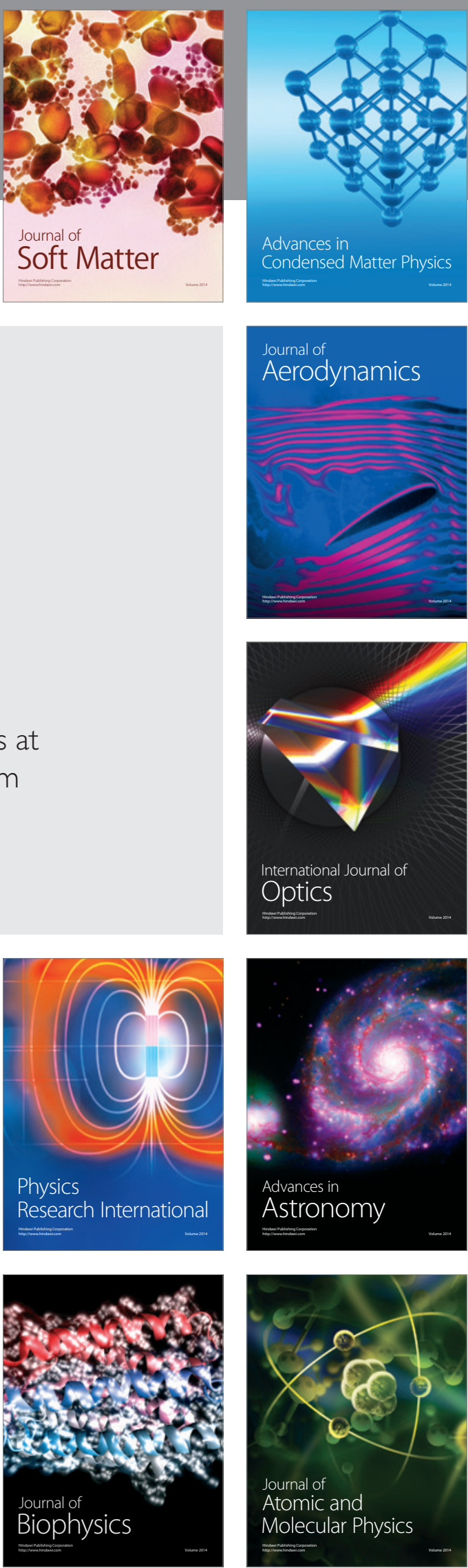